\title{
Revisiting mitochondrial diagnostic criteria in the new era of genomics
}

\author{
Peter Witters, MD, PhD ${ }^{1}$, Ann Saada, MD, $\mathrm{PhD}^{2,3}$, Tomas Honzik, MD, PhD ${ }^{4}$, Marketa Tesarova, $\mathrm{PhD}^{4}$, \\ Stephanie Kleinle, $\mathrm{PhD}^{5}$, Rita Horvath, MD, $\mathrm{PhD}^{6}$, Amy Goldstein, MD, PhD ${ }^{7}$ and Eva Morava, MD, $\mathrm{PhD}^{1,7}$
}

Purpose: Diagnosing primary mitochondrial diseases (MDs) is challenging in clinical practice. The mitochondrial disease criteria (MDC) have been developed to quantify the clinical picture and evaluate the probability of an underlying MD and the need for a muscle biopsy. In this new genetic era with next-generation sequencing in routine practice, we aim to validate the diagnostic value of MDC.

Methods: We retrospectively studied MDC in a multicenter cohort of genetically confirmed primary MD patients.

Results: We studied 136 patients (61 male, 91 nuclear DNA (nDNA) mutations). Forty-five patients (33\%) had probable MD and 69 (51\%) had definite MD according to the MDC. A muscle biopsy was performed in 63 patients (47\%). Patients with nDNA mutations versus mitochondrial DNA mutations were younger $(6.4 \pm 9.7$ versus $19.5 \pm 17.3 \mathrm{y})$ and had higher MDC
$(7.07 \pm 1.12 / 8$ versus $5.69 \pm 1.94 / 8)$. At a cutoff of $6.5 / 8$, the sensitivity to diagnose patients with nDNA mutations is $72.5 \%$ with a positive predictive value of $69.5 \%$. In the nDNA mutation group, whole-exome sequencing could diagnose patients with lower scores (MDC $(6.84 \pm 1.51 / 8)$ compared to Sanger sequencing MDC $(7.44 \pm 1.13 / 8, P=0.025))$. Moreover $7 / 8$ patients diagnosed with possible MD by MDC were diagnosed by whole-exome sequencing.

Conclusion: MDC remain very useful in the clinical diagnosis of $\mathrm{MD}$, in interpreting whole-exome results and deciding on the need for performing muscle biopsy.

Genet Med advance online publication 26 October 2017

Key Words: diagnosis; mitochondrial disease; mitochondrial disease criteria; muscle biopsy; whole-exome sequencing

\section{INTRODUCTION}

Mitochondrial disease is the most frequent, often devastating, neurometabolic disease, with an estimated incidence of $\sim 1 / 5,000$ births. ${ }^{1}$ This is probably an underestimate as the diagnosis is certainly not straightforward. ${ }^{2-4}$ Mutations in the same mitochondrial disease gene-which can be either nuclear DNA (nDNA) or mitochondrial DNA (mtDNA) encoded-can give rise to different clinical phenotypes. On the other hand the same clinical picture can be caused by a myriad of genes affecting mitochondrial function, or involved in totally different pathways, like glycosylation, neurotransmission, or development. ${ }^{5}$

In some patients there is a clinically recognizable syndrome (for instance, mitochondrial myopathy, encephalopathy, lactic acidosis, and stroke-like episodes (MELAS; MIM 540000) or myoclonic epilepsy associated with ragged-red fibers (MERRF; MIM 545000) and the genetic analysis can be guided by the clinical symptoms. However, in the majority of patients, the clinical phenotype is less definitive. To this extent, the mitochondrial disease criteria (MDC) have been developed to facilitate mitochondrial diagnostics. ${ }^{5,6}$ Historically, two different mitochondrial disease criteria have been published. In 2002 Bernier et al. ${ }^{7}$ established mitochondrial diagnostic criteria in children based on a previously validated adult mitochondrial score. The goal of this scoring system was to establish the complex diagnosis of clinical and biochemically proven mitochondrial disease. The score developed by Wolf et al. ${ }^{6}$ had a different goal. It was established to help the clinician during the diagnostic process to decide on the need for surgical muscle biopsy and enzyme complex measurement. This score was further simplified as a bedside instrument for screening for a suspected mitochondrial disorder. ${ }^{5}$ It is this latter score that we study in the current manuscript. This composite score helps to quantify the probability that a patient has an oxidative phosphorylation (OXPHOS) defect-related disease. It was also instrumental in decision making on performing more invasive investigations, such as a muscle biopsy. It is tailored for children in whom symptoms are still evolving into a more characteristic phenotype. ${ }^{5}$ However, just like muscle biopsies, the MDC

\footnotetext{
${ }^{1}$ Department of Paediatrics and Metabolic Center, University Hospitals Leuven, Department of Development and Regeneration, KU Leuven, Leuven, Belgium; ${ }^{2}$ Monique and Jacques Roboh Department of Genetic Research, Hadassah-Hebrew University Medical Centre, Jerusalem Israel; ${ }^{3}$ The Department of Genetic and Metabolic Diseases, Hadassah-Hebrew University Medical Centre, Jerusalem Israel; ${ }^{4}$ Department of Pediatrics, First Faculty of Medicine, Charles University and General University Hospital, Prague, Czech Republic; ${ }^{5}$ Medical Genetics Centre, Munich, Germany; ${ }^{6}$ Institute of Genetic Medicine, Newcastle University, Newcastle upon Tyne, UK; ${ }^{7}$ Tulane University Medical School, Hayward Genetics Center, New Orleans, Louisiana, USA. Correspondence: Peter Witters (peter.witters@uzleuven.be) or Eva Morava (emoravakozicz@tulane.edu) The first three authors contributed equally to this work.

The last two authors contributed equally to this work. 
cannot differentiate between primary and secondary respiratory chain involvement. ${ }^{6}$

Overall $\sim 5 \%$ of the human genome is predicted to be involved in mitochondria (MitoCarta ${ }^{8}$ ). Currently, more than 250 genes are known to be associated with mitochondrial disease, but this list is growing rapidly in an era of new genetic techniques, such as next-generation sequencing (NGS). ${ }^{9-16}$ Interestingly, in patients diagnosed with a mitochondrial disease (compatible clinical signs and symptoms and a confirmed mitochondrial OXPHOS complex deficiency), the success of genetic diagnostics by NGS is between 16 and $60 \%$ depending on patient selection and diagnostic pipeline used. ${ }^{9-16}$

Over the past several years, the development of novel diagnostic techniques and our growing understanding of mitochondrial pathophysiology altered the clinical diagnostic approach in suspected mitochondrial cases. With the increased availability of NGS, mainly whole-exome sequencing (WES) or targeted panels, most diagnostic centers changed the diagnostic strategy in suspected mitochondrial disease. ${ }^{17}$ The current approach to mitochondrial diagnostics, even by a low suspicion, is to rule out mtDNA mutations by direct sequencing, followed by (or in parallel with) NGS panels or $\mathrm{WES}^{18}$ looking at nDNA. Therefore, many clinicians practicing mitochondrial medicine question whether muscle biopsy is still the gold standard for the diagnosis of mitochondrial disease. Moreover, defects in mitochondrial maintenance, fusion/fission, translation, transcription, or abnormalities in mitochondrial membrane integrity or transport have taught us that some of these disorders do not always present with significant OXPHOS deficiencies and may present with normal muscle biochemistry and histology results. ${ }^{2}$ Nevertheless, the identification of new mutations often requires extensive research to prove its pathogenicity, and it is needless to point out that these research costs are not included in the cost of WES or covered by any health insurance. ${ }^{19}$

WES provided us with many new genes and novel phenotypes, and our diagnostic strategy has changed profoundly. One should raise the question whether the MDC still provide us with sufficient guidance in our diagnostic choices, and if they are reliable enough to point toward this "new cohort" of mitochondrial patients. The aim of our study is to review a cohort of patients with molecularly confirmed disease in several international centers and evaluate the MDC's ability to predict disease.

\section{Patients}

\section{MATERIALS AND METHODS}

In this retrospective analysis we included patients with genetically confirmed primary mitochondrial diseases diagnosed between January 2010 and December 2015 from seven metabolic centers. None of the patients were diagnosed postmortem (Supplementary Data 1 online). Current research is in accordance with the ethical standards of the committee on human experimentation and with the Helsinki Declaration.

\section{Methods}

All participating centers used either mtDNA analysis (sequencing, restriction fragment length polymorphism, mtDNA long-range polymerase chain reaction, Southern blot) followed by either WES or nDNA mitochondrial gene panels or direct Sanger sequencing of nuclear genes based on clinical findings.

Data collected from electronic medical records included age, gender, survival, (age of death), specific clinical features, family history, biochemical testing and imaging data used for obtaining the MDC (data at the time of the diagnosis ${ }^{5}$ ), the genetic technique used, the genetic diagnosis, the histology, and OXPHOS complex measurements (if a muscle biopsy was performed). In two patients with complex II deficiency OXPHOS complex measurements on lymphoblasts were performed.

The MDC were calculated adding up the scores of clinical (muscle, central nervous system, and multisystem involvement), biochemical and imaging results, and in case of biopsy the results of histology, as described previously. ${ }^{5}$ A score of 1 indicates unlikely mitochondrial disorder, score 2-4: possible mitochondrial disorder, score 5-7 probable mitochondrial disorder, and score $\geq 8$ definite mitochondrial disorder (Supplementary Data 2).

\section{Statistics \\ See Supplementary Data 1. \\ RESULTS}

\section{Population}

We included 136 patients (61 male, 75 female) with a mean age of $10.7 \pm 14.1$ years (median 3.75 years, range 0-68 years). At the time of this manuscript, 50 (37\%) of these patients had died. Pathogenic nDNA variants were detected in $91(67 \%)$ cases. Forty-five patients $(33 \%)$ had a pathogenic mtDNA variant $(n=39)$ or mtDNA deletion $(n=6)$. In 18 individuals with pathogenic mtDNA variants, a mutation in MT-TL1 was detected. Other pathogenic mtDNA variants were MT-ATP6 $(n=4), M T-N D 1(n=2), M T-N D 3(n=1)$, MT-ND5 $(n=6)$, MT-ND6 $(n=2), M T-R N R 1 \quad(n=2)$, MT-TK $(n=1), M T-T S 1 \quad(n=2)$, and MT-TW $(n=1)$. Pathogenic nDNA variants were detected in AARS2 $(n=3), \operatorname{ADCK} 3(n=1), \operatorname{AIFM1}(n=3)$, C10ORF2 $(n=1)$, C12ORF65 $(n=4)$, COX10 $(n=1)$, COX6B1 $(n=1)$, DARS2 $(n=2)$, DGUOK $(n=1)$, DNM1L $(n=1), \operatorname{EARS} 2(n=1)$, ELAC2 $(n=3)$, ETHE1 $(n=1)$, FDX1L $(n=1)$, GFM1 $(n=1), \quad$ GTPBP3 $(n=1), \quad M P V 17 \quad(n=2), \quad$ MTFMT $(n=1), \quad$ MTO1 $(n=1), \quad$ NDUFAF2 $(n=1)$, NDUFAF5 $(n=3), \quad$ NDUFB3 $(n=1), \quad$ NDUFS1 $(n=1), \quad$ NDUFS 8 $(n=1)$, NDUFV1 $(n=1)$, OPA1 $(n=3)$, PDHA1 $(n=2)$, POLG $(n=11)$, PUS1 $(n=2)$, RARS2 $(n=1)$, RMND1 $(n=2), \operatorname{SCO} 2(n=5), \operatorname{SDHAF} 1(n=2), \operatorname{SLC} 25 A 1(n=2)$, SUCLG1 $(n=2)$, SURF1 $(n=5)$, TK2 $(n=1)$, TMEM70 $(n=9), \operatorname{TRAP} 1(n=1), \operatorname{TSFM}(n=2)$, and TYMP $(n=3)$.

Composite MDC score was calculated for all patients (Supplementary Data 3). We found 103 patients (75\%) with 
Table 1 Mitochondrial score nuclear versus mitochondrial DNA

Mitochondrial disease criteria

Muscular presentation

Ophthalmoplegia (score 2)

Facies myopathica

Exercise intolerance

Muscle weakness

Rhabdomyolysis

CNS presentation

Developmental delay

Loss of skills

Stroke-like episodes

Migraine

Seizures

Myoclonus

Cortical blindness

Pyramidal signs

Extrapyramidal signs

Brain stem involvement

Multisystem disease

Hematology

Gl tract

Endocrine/growth

Heart

Kidney

Vision

Hearing

Neuropathy

Recurrent/familial

Elevated lactate (score 2

Elevated L/P ratio

Elevated alanine (score 2)

Elevated CSF lactate (score 2)

Elevated CSF protein

Elevated CSF alanine (score 2)

Krebs cycle intermediates (score 2)

Ethylmalonic aciduria

Stroke-like picture on MRI

Leigh syndrome on MRI (score 2)

Elevated lactate on MRS

Morphology

Ragged red/blue fibers (score 4)

COX-negative fibers (score 4)

Reduced COX staining (score 4)

Reduced SDH staining

SDH-positive blood vessels (score 2)

Abnormal mitochondria on EM (score 2)
Abnormal EMG

Metabolic/imaging studies
nDNA $^{a}$

mtDNA

$11 / 91$

$12 / 91$

20/90

$78 / 91$

2/91

$10 / 56$

76/91

42/91

$6 / 91$

$5 / 91$

$33 / 91$

$14 / 91$

15/91

$31 / 91$

$38 / 91$

20/90

4/91

$31 / 91$

42/91

$30 / 91$

$7 / 91$

$32 / 91$

13/91

$14 / 89$

28/91

$76 / 87$

28/60

29/80

$30 / 57$

$14 / 53$

$15 / 49$

29/78

$5 / 77$

$5 / 77$

$33 / 77$

$14 / 63$

$1 / 28$

$7 / 28$

$4 / 27$

$0 / 26$

$7 / 26$

$5 / 23$

mENA

$8 / 45$

$0 / 45$

$10 / 45$

$21 / 45$

$0 / 45$

$5 / 42$

$12 / 45$

$10 / 45$

$8 / 45$

$8 / 45$

$15 / 45$

$5 / 45$

$1 / 45$

$3 / 45$

4/45

0/45

$1 / 45$

$4 / 45$

$17 / 45$

$9 / 45$

2/45

$16 / 45$

$24 / 45$

$6 / 45$

$8 / 45$

$31 / 36$

$7 / 18$

$13 / 20$

$16 / 17$

$6 / 15$

$7 / 10$

$2 / 21$

$1 / 21$

$7 / 21$

$7 / 23$

$2 / 21$

$6 / 20$

$9 / 20$

$5 / 18$

$0 / 15$

$11 / 15$

2/18
Total

nDNA versus mtDNA ( $P$ value)

$\begin{array}{cr}19 / 136 & 0.433 \\ 12 / 136 & 0.009^{c} \\ 30 / 135 & 1.000 \\ 99 / 136 & <0.001^{c} \\ 2 / 136 & 1.000 \\ 15 / 98 & 0.573\end{array}$

CNS, central nervous system; CSF, cerebrospinal fluid; COX, cytochrome c oxidase; EM, electron microscopy; EMG, electromyography; GI, gastrointestinal; L/P, lactate/ pyruvate; MRI, magnetic resonance imaging; MRS, magnetic resonance spectroscopy; mtDNA, mitochondrial DNA; NA, not available; nDNA, nuclear DNA; SDH, succinate dehydrogenase; TA, tricarbon acid.

Fischer's exact test 2-sided was used.

a Number of patients with the sign present/number of patients with the sign checked. ${ }^{b} P<0.05,{ }^{c} P<0.01$. 


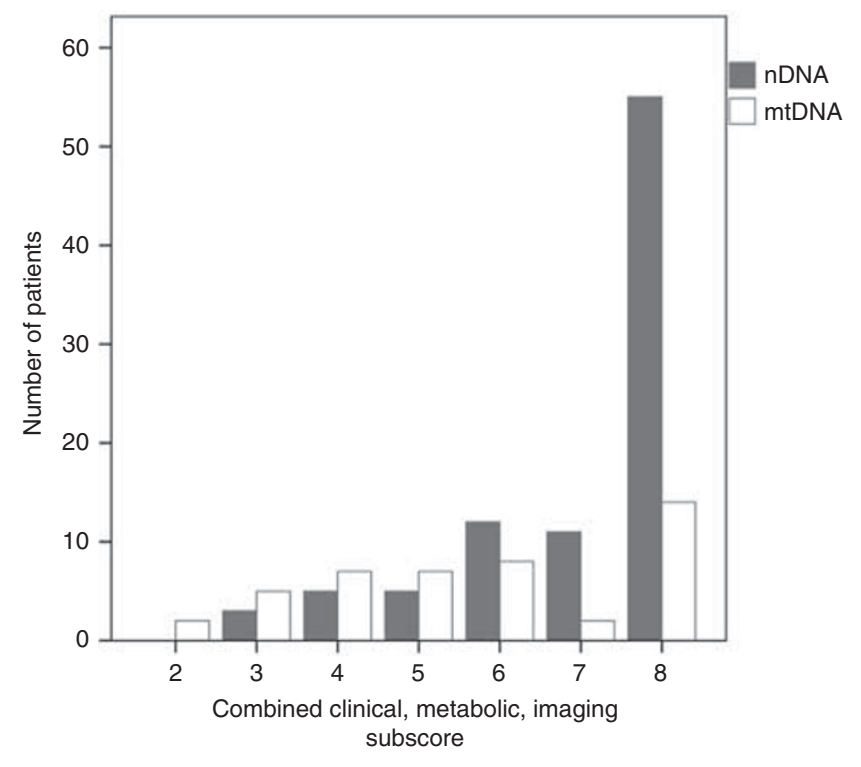

Figure 1 Mitochondrial score nuclear versus mitochondrial DNA. mtDNA, mitochondrial DNA; nDNA, nuclear DNA.

a maximal clinical subscore (4/4), while 77 (56\%) had a maximal metabolic and imaging subscore (4/4). Based on these scores, 45 patients $(33 \%)$ have probable mitochondrial disease (clinical/metabolic/imaging score: 5-7/8) and 69 patients (51\%) have the maximal subscore (8/8) indicating definite mitochondrial disease (for a more detailed description see Table 1 and Figure 1). In 63 (47\%) patients a muscle biopsy was performed as part of the diagnostics, which included 3 patients with mtDNA mutations to confirm a genetic diagnosis. In 61 patients, the enzymatic activities of the mitochondrial OXPHOS complexes were measured in the clinical setting. In 5 of these, there were no abnormalities although these patients have pathogenic variants that were reported previously. In 21 cases there was a single complex deficiency, in 26 cases two complexes, in 6 cases three, and in 3 cases four complexes were deficient. Complexes I, II, III, IV, and $\mathrm{V}$ were deficient in $66 \%, 15 \%, 26 \%, 54 \%$, and $8 \%$ of patients, respectively. Complex III deficiency was more prevalent among patients with pathogenic nDNA variants $(10 / 20$ versus $6 / 41, P=0.005)$ and complex IV deficiency among patients with mtDNA abnormalities (30/41 versus $3 / 20, P<0.001)$.

\section{mtDNA versus nDNA abnormalities}

Patients with nDNA mutations were significantly younger $(6.4 \pm 9.7$ years versus $19.5 \pm 17.3$ years, $P<0.001)$. Overall, they showed a higher score for muscle symptoms (subscore of $1.33 \pm 0.65 / 2$ versus $0.98 \pm 0.81 / 2, P=0.014$ ), for central nervous system findings (subscore of $1.60 \pm 0.70 / 2$ versus $1.02 \pm 0.87 / 2, P=<0.001$ ), and more metabolic and imaging abnormalities $(3.30 \pm 1.1 / 4$ versus $2.6 \pm 1.2 / 4$, $P=0.001)$ resulting in a higher clinical/metabolic/imaging subscore $(7.07 \pm 1.12 / 8$ versus $5.69 \pm 1.94 / 8, P<0.001)$ (see
Figure 1). Several items from the MDC were more frequent in the patients with nDNA mutations, such as myopathic facies, muscle weakness, developmental delay, regression cortical blindness, pyramidal signs, extrapyramidal signs, brain stem involvement, gastrointestinal tract involvement, and urinary Krebs cycle intermediates excretion. The features more prevalent in the patients with mtDNA mutation include migraine, hearing deficit, elevated blood alanine, elevated cerebrospinal fluid lactate, elevated cerebrospinal fluid alanine, and stroke-like picture on magnetic resonance image (see Table 1). The presence of brain stem involvement or the absence of a hearing deficit could predict the presence of an nDNA mutation in $78.5 \%$ of cases.

The area under the receiver operating characteristic curve of the MDC for the prediction of an nDNA mutation is 0.702 (95\% confidence interval: $0.603-0.800, P<0.001$ ). At the point of best cutoff (6.5) this has a sensitivity and specificity of $72.5 \%$ and $64.4 \%$ respectively. In our cohort this yields a positive predictive value of $69.5 \%$ and a negative predictive value of $38.9 \%$.

\section{Genetic techniques used}

Looking at the 91 patients with nDNA mutations, the final molecular diagnosis was made by (multiple) Sanger sequencing of single genes in $34(37 \%)$ and by WES or a targeted mitochondrial gene panel in 57 (63\%).

In patients with cortical blindness, the diagnosis was more often reached through WES/gene panel (13/57 versus 2/34, $P=0.043)$. Biochemical changes, on the other hand, were more often encountered in patients with a diagnosis through Sanger sequencing: elevated blood lactate/pyruvate ratio $(17 / 20$ versus $11 / 40, \quad P<0.001)$, elevated blood alanine $(19 / 27$ versus $10 / 53, P<0.001)$, increased urinary Krebs cycle intermediate excretion $(17 / 30$ versus $12 / 48, P=0.008)$.

While there was no difference in the clinical score, the combined metabolic findings and imaging subscore was higher in the patients with Sanger sequencing used as a genetic tool $(3.560 \pm 0.991 / 4, n=34$ versus $3.140 \pm 1.172 / 4$, $n=57 ; P=0.046)$. Similarly, the combined clinical/metabolic/imaging score was significantly lower in the patients with gene panel/WES diagnosis compared to Sanger sequencing $(6.842 \pm 1.512 / 8, \quad n=57 \quad$ versus $7.441 \pm 1.133 / 8$, $n=34$; $P=0.025$ ) (see Figure 2). Moreover, looking at the patients with a low score $(<5 / 8$, i.e., possible but not probable mitochondrial disorder), only one patient (NDUFAF5 mutation) out of eight had a diagnosis through Sanger sequencing after linkage analysis. The other patients had mutations in AARS2, AIFM1, DNM1L, ELAC2, GTPBP3, $M T F M T$, and OPA1. In all of these patients in whom a muscle biopsy was performed (7/8 patients, not in OPA1 patient), decreased OXPHOS complex activities were observed in concert with a mitochondrial disease.

Muscle biopsies were performed in the same percentage of patients in both diagnostic groups (Sanger 14/34 versus gene panel/WES 28/57, $P=n s$ ). A complex I/IV deficiency was more prevalent in the gene panel/WES group (21/28 and 
$25 / 28$ versus $4 / 13$ and $5 / 13 ; P=0.014$ and 0.001 , respectively) and less often a complex $\mathrm{V}$ deficiency (and $0 / 28$ versus $4 / 13, P=0.007$ ); while histologic findings were comparable. Overall the activities of multiple complexes were more decreased in the gene panel/WES group (1.964 \pm 0.838 complexes abnormal versus $1.231 \pm 0.838$ complex; $P=0.007)$.

There was no difference with regard to the patient's survival and the genetic technique used.

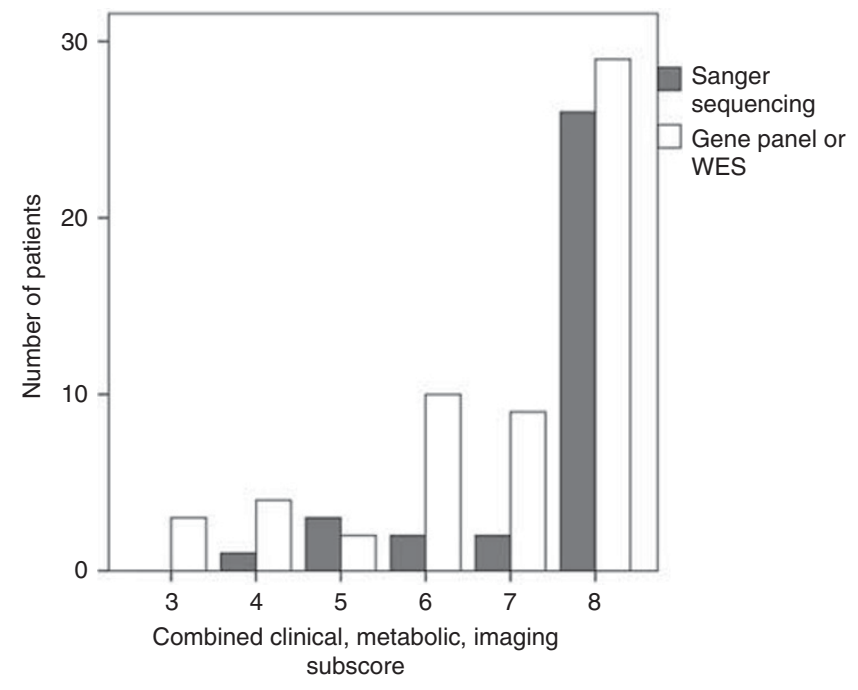

Figure 2 Mitochondrial score Sanger sequencing versus wholeexome sequencing/gene panel. WES, whole-exome sequencing.

\section{MDC distribution}

Looking at the MDC in this entire cohort of genetically proven mitochondrial disease, 51\% (69/136) had a maximal mitochondrial score predicting a definite mitochondrial disorder, 33\% $(45 / 136)$ had a probable mitochondrial disorder, and $16 \%$ $(22 / 136)$ had a possible mitochondrial disorder. In patients with nDNA mutations (for whom the traditional genetic diagnostic approach can be very elaborate) the score showed more often a definite mitochondrial disease (60\%) and less often probable $(31 \%)$ or possible $(9 \%)$ compared to the patients with mtDNA abnormalities $(31 \%, 38 \%$, and $31 \%$ respectively, $P<0.001$ ) (see Figure 1). In these patients, when Sanger sequencing was used, a higher mitochondrial score was obtained $(7.441 \pm 1.133 / 8, n=34$ versus $6.842 \pm 1.512 / 8$, $n=57 ; P=0.025$ ) (see Figure 2). This means that these patients diagnosed by Sanger sequencing had a more often definite diagnosis $(76 \%)$ rather than a probable $(21 \%)$ or possible $(3 \%)$ mitochondrial disease compared to the patient with WES (51\%, 37\% and $12 \%$ respectively, $P=0.05)$.

\section{DISCUSSION}

In the present work we studied the use and utility of the MDC in an era in which new high-throughput genetic techniques (mtDNA sequencing, WES) have come to routine clinical practice. Despite these technical advances, clinical, biochemical, and imaging findings remain very useful in initiating specific molecular diagnostics to establish a mitochondrial diagnosis. The practical use of the MDC and the integration with the genetic results is illustrated in the diagnostic flowchart (see Figure 3).

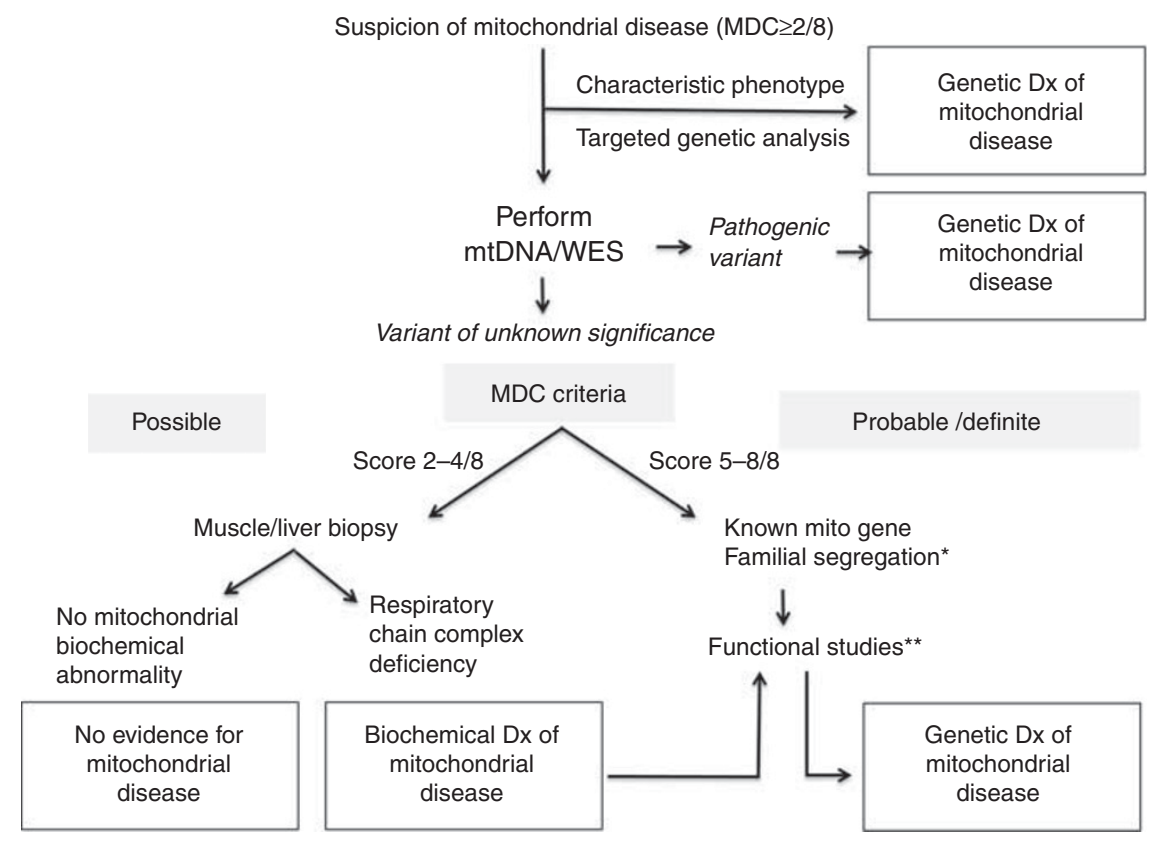

Figure 3 Proposed diagnostic flowchart. *Familial segregation: in the case of mtDNA changes this takes into account the degree of heteroplasmy, tissue variation, etc. ${ }^{*}$ Functional studies to prove the pathogenicity include enzyme expression and complementation studies, complexomics, etc. MDC, mitochondrial disease criteria; mtDNA, mitochondrial DNA; WES, whole-exome sequencing. 
Several mitochondrial syndromes are recognizable based on clinical picture, biochemistry, or imaging findings that can lead straight to the targeted analysis and subsequent genetic diagnosis. In our current series, this was the case for most patients with MELAS; MERRF; neuropathy, ataxia, and retinitis pigmentosa (NARP); Kearns-Sayre syndrome; TMEM70 (encephalocardiomyopathy, 3-methylglutaconaciduria, hypospadias); SURF1 (Leigh syndrome); SCO2 (fatal infantile encephalocardiomyopathy); PDHA1 (pyruvate dehydrogenase deficiency: encephalomyopathy and Leigh syndrome); TYMP (mitochondrial neurogastrointestinal encephalopathy syndrome (MINGIE); POLG (Alpers syndrome: encephalohepatopathy); and PUS1 (myopathy (hypotonia), lactic acidosis, and sideroblastic anemia). ${ }^{20}$ However, in some patients with recognizable syndromes such as OPA1 (optic atrophy), DARS2 (leukoencephalopathy with brain stem and spinal cord involvement and lactate elevation), and SUCLG1 (encephalomyopathy, fatal infantile acidosis with methylmalonic aciduria) the diagnosis was made through WES.

Table 2 Mitochondrial disease criteria ${ }^{5}$

\section{Features}

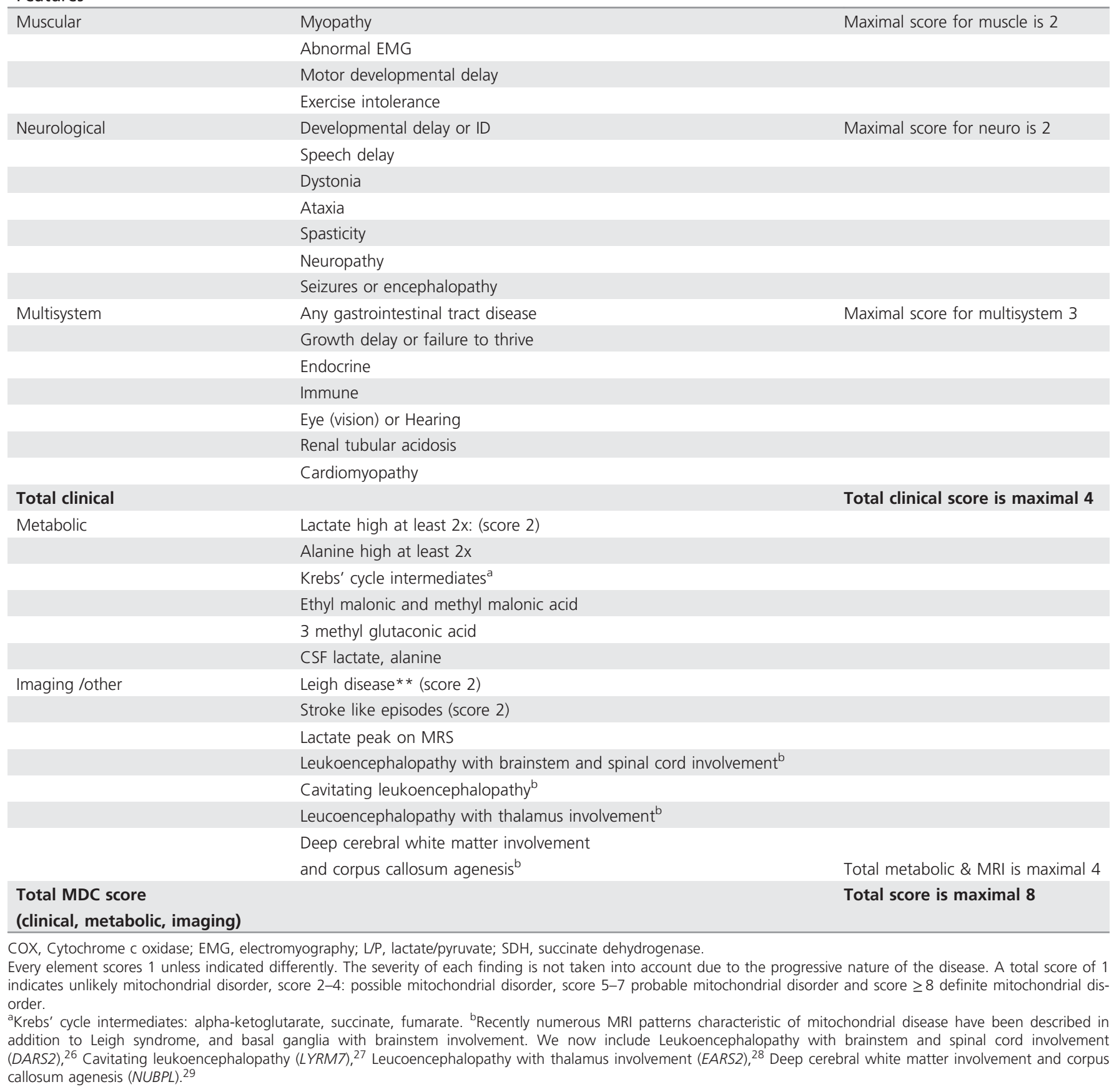


Moreover, the clinical phenotyping with the use of the MDC provides support for the interpretation of WES findings. If in a patient with a high mitochondrial score WES analysis reveals compatible mutations in mitochondrial genes, this is likely to be a pathogenic finding. Indeed, in the patients in whom the diagnosis was made by WES $88 \%$ of patients had a definite or probable score. It has been previously shown that a high MDC score increases the odds of finding a genetic diagnosis by WES. ${ }^{15,21}$ Of note, in our study, as many as $51 \%$ had a definite mitochondrial disease as assessed by the MDC.

The MDC can also help to orient the further genetic analysis. Indeed, in our present cohort, the MDC score was significantly higher in the patients with nDNA mutations $(7.07 \pm 1.12 / 8$ versus $5.69 \pm 1.94 / 8, P<0.001$ ). A score $>6 / 8$ has a positive predictive value of $70 \%$ for an nDNA mutation in this study cohort. Traditionally, the first step in the genetic analysis is the study of the mtDNA in blood (preferably through NGS), and if needed, in multiple symptomatic tissues. ${ }^{17}$ With the increased availability of WES and in patients with a high MDC score, mtDNA screening is not necessarily performed prior to WES (unless there is a clear maternal inheritance). The use of WES combining mtDNA and nDNA will probably be replaced in the future by combined techniques that can reliably detect mtDNA mutations. ${ }^{22}$

Nevertheless, in this new genetic era, looking at the patients with nDNA mutations leading to a mitochondrial disease, we observed a lower score in the patients diagnosed through WES compared to Sanger sequencing $(6.842 \pm 1.512 / 8$, $n=57$ versus $7.441 \pm 1.133 / 8, n=34 ; P=0.025)$ as milder, less clear-cut phenotypes were diagnosed. This leads to a decrease in sensitivity as more patients with lower scores are shown to have a mitochondrial disease by WES. Indeed, looking at these lower scores there were $12 \%$ of the patients in the WES group who had only a possible MDC score, compared with only $3 \%$ in the Sanger group. Using WES, seven patients $(12 \%)$ were identified (of whom three had a clinical/metabolic/imaging score of $3 / 8$ and four with a score of 4/8) while with Sanger sequencing (+ linkage analysis) only one patient (3\%) with a score of $4 / 8$ was found. Thus, we propose that in this new era of NGS genetic analysis should not be restricted to patients with a probable or definite mitochondrial disease (MDC score $\geq 5 / 8$ ), because most likely some of the primary OXPHOS diagnoses will be missed.

The primary choice of tissue for sequencing is blood. Muscle is primarily used for ruling out mtDNA deletions/ depletion, based on clinical suspicion if it was not detected in blood. The role of muscle biopsy used to be central, in fact the "gold standard," in the diagnosis of mitochondrial diseases. We have recently suggested a new diagnostic algorithm, placing this as the final examination after the genetic investigations. Muscle biopsies are invasive investigations that often require general anesthesia, which can be detrimental for mitochondrial patients, and results do not differentiate between primary versus secondary mitochondrial dysfunction. ${ }^{23}$ Based on current research we propose the following indications: if there is "probable" MDC and WES gives pathogenic variants in genes associated with mitochondrial disease, which suits the phenotype and segregates within the family, the diagnosis is made and the muscle biopsy is not necessary. A muscle (and/or liver) biopsy should (only) be performed in routine analysis for mitochondrial disease when the diagnosis cannot be confirmed with DNA testing. ${ }^{17,18}$ In our cohort this invasive investigation could be omitted in $54 \%$ of patients, a number that will hopefully increase in the future. However, in case of a high prediction of pathogenicity in a new variant/candidate gene, a muscle biopsy with histology, OXPHOS measurements, and other functional evaluations can be helpful in the interpretation of the data.

Recently, there have been great improvements in magnetic resonance image pattern recognition (other than Leigh disease) in diagnosing mitochondrial diseases. We suggest to include these in the MDC score to improve sensitivity (Table 2). This could even further decrease the need for muscle biopsy.

\section{Conclusion}

We have shown that the MDC remain very useful in the clinical diagnosis of MD. They can help the diagnostic process, interpreting whole-exome results and deciding on performing muscle biopsy in selected cases.

In this new genetic era, we are also diagnosing patients with lower MDC scores as primary mitochondrial diseases. Early diagnosis is essential, and we anticipate that this patient group will soon benefit from emerging therapies. ${ }^{24,25}$

\section{SUPPLEMENTARY MATERIAL}

Supplementary material is linked to the online version of the paper at http://www.nature.com/gim

\section{ACKNOWLEDGMENTS}

T.H. and M.T. were supported by the Ministry of Health of the Czech Republic (MZ CR AZV 16-32341A), the Grant Agency of the Czech Republic (14-36804G), and by Charles University in Prague PRVOUK P24/LF1/3. E.M. was supported by the Hayward Genetics Trustees subsidy. P.W. is supported by the Clinical Research Foundation of University Hospitals Leuven, Leuven, Belgium. A.S. was supported by the Pakula family via American Friends of the Hebrew University. Orly Elpeleg is acknowledged for contributing to the WES data.

\section{DISCLOSURE}

The authors declare no conflict of interest.

\section{REFERENCES}

1. Schaefer AM, Taylor RW, Turnbull DM, Chinnery PF. The epidemiology of mitochondrial disorders-past, present and future. Biochim Biophys Acta 2004;1659:115-120.

2. DiMauro S, Schon EA, Carelli V, Hirano M. The clinical maze of mitochondrial neurology. Nat Rev Neurol 2013;9:429-444.

3. Lightowlers RN, Taylor RW, Turnbull DM. Mutations causing mitochondrial disease: what is new and what challenges remain? Science 2015;349:1494-1499. 
4. Chinnery PF. Mitochondrial disease in adults: what's old and what's new? EMBO Mol Med 2015;7:1503-1512.

5. Morava E, van den Heuvel L, Hol F, et al. Mitochondrial disease criteria: diagnostic applications in children. Neurology 2006;67:1823-1826.

6. Wolf NI, Smeitink JA. Mitochondrial disorders: a proposal for consensus diagnostic criteria in infants and children. Neurology 2002;59: 1402-1405.

7. Bernier FP, Boneh A, Dennett X, Chow CW, Cleary MA, Thorburn DR. Diagnostic criteria for respiratory chain disorders in adults and children. Neurology 2002;59:1406-1411.

8. Calvo SE, Clauser KR, Mootha VK. MitoCarta2.0: an updated inventory of mammalian mitochondrial proteins. Nucleic Acids Res 2016;44: D1251-D1257.

9. Shamseldin HE, Alshammari M, Al-Sheddi T, et al. Genomic analysis of mitochondrial diseases in a consanguineous population reveals novel candidate disease genes. J Med Genet 2012;49:234-241.

10. Calvo SE, Compton AG, Hershman SG, et al. Molecular diagnosis of infantile mitochondrial disease with targeted next-generation sequencing. Sci Trans/ Med 2012;4:118ra110.

11. Koene S, Rodenburg RJ, van der Knaap MS, et al. Natural disease course and genotype-phenotype correlations in complex I deficiency caused by nuclear gene defects: what we learned from 130 cases. J Inherit Metab Dis 2012;35:737-747.

12. Taylor RW, Pyle A, Griffin H, et al. Use of whole-exome sequencing to determine the genetic basis of multiple mitochondrial respiratory chain complex deficiencies. JAMA 2014;312:68-77.

13. Kohda M, Tokuzawa $Y$, Kishita $Y$, et al. A comprehensive genomic analysis reveals the genetic landscape of mitochondrial respiratory chain complex deficiencies. PLoS Genet 2016;12:e1005679.

14. Neveling K, Feenstra I, Gilissen C, et al. A post-hoc comparison of the utility of Sanger sequencing and exome sequencing for the diagnosis of heterogeneous diseases. Hum Mutat 2013:34:1721-1726.

15. Wortmann SB, Koolen DA, Smeitink JA, van den Heuvel L, Rodenburg RJ. Whole exome sequencing of suspected mitochondrial patients in clinical practice. J Inherit Metab Dis 2015;38:437-443.

16. Legati $A$, Reyes $A$, Nasca $A$, et al. New genes and pathomechanisms in mitochondrial disorders unraveled by NGS technologies. Biochim Biophys Acta 2016;1857:1326-1335.
17. Parikh S, Goldstein A, Koenig MK, et al. Diagnosis and management of mitochondrial disease: a consensus statement from the Mitochondrial Medicine Society. Genet Med 2015;17:689-701.

18. Morava E, Brown GK. Next generation mitochondrial disease: change in diagnostics with eyes on therapy. J Inherit Metab Dis 2015;38: 387-388.

19. Saada A. Mitochondria: mitochondrial OXPHOS (dys) function ex vivothe use of primary fibroblasts. Int J Biochem Cell Biol 2014;48:60-65.

20. Honzik T, Tesarova M, Magner $M$, et al. Neonatal onset of mitochondrial disorders in 129 patients: clinical and laboratory characteristics and a new approach to diagnosis. J Inherit Metab Dis 2012;35:749-759.

21. Pronicka E, Piekutowska-Abramczuk D, Ciara E, et al. New perspective in diagnostics of mitochondrial disorders: two years' experience with whole-exome sequencing at a national paediatric centre. J Trans/ Med 2016;14:174.

22. Griffin HR, Pyle A, Blakely EL, et al. Accurate mitochondrial DNA sequencing using off-target reads provides a single test to identify pathogenic point mutations. Genet Med 2014;16:962-971.

23. Rivera-Cruz B. Mitochondrial diseases and anesthesia: a literature review of current opinions. AANA J 2013;81:237-243.

24. Koopman WJ, Beyrath J, Fung CW, et al. Mitochondrial disorders in children: toward development of small-molecule treatment strategies. EMBO Mol Med 2016;8:311-327.

25. Rahman S. Emerging aspects of treatment in mitochondrial disorders. J Inherit Metab Dis 2015;38:641-653.

26. Steenweg ME, Pouwels PJ, Wolf NI, van Wieringen WN, Barkhof F \& van der Knaap MS. Leucoencephalopathy with brainstem and spinal cord involvement and high lactate: quantitative magnetic resonance imaging. Brain 2011;134:3333-3341.

27. Dallabona C, Abbink TE, Carrozzo R, et al. LYRM7 mutations cause a multifocal cavitating leukoencephalopathy with distinct MRI appearance. Brain :2016;139:782-794.

28. Oliveira R, Sommerville EW, Thompson K, et al. Lethal Neonatal LTBL Associated with Biallelic EARS2 Variants: Case Report and Review of the Reported Neuroradiological Features. JIMD reports 2016.

29. Kevelam SH, Rodenburg RJ, Wolf NI, et al. NUBPL mutations in patients with complex I deficiency and a distinct MRI pattern. Neurology 2013;80: 1577-1583. 